\title{
Role of depth of response and MTHFR genotype as predictors of fluorouracil rechallenge therapy for refractory metastatic colorectal cancer
}

\author{
KA-RHAM KIM ${ }^{1}$, JUNG-HWAN YOON ${ }^{1}$, HYUN-JEONG SHIM ${ }^{1}$, JUN-EUL HWANG ${ }^{1}$, \\ WOO-KYUN BAE ${ }^{1}$, IK-JOO CHUNG ${ }^{1}$, HEE-NAM KIM ${ }^{2}$, MIN-HO SHIN ${ }^{2}$ and SANG-HEE CHO ${ }^{1}$ \\ Departments of ${ }^{1}$ Hemato-Oncology and ${ }^{2}$ Preventive Medicine, \\ Chonnam National University Medical School, Gwangju 61186, Republic of Korea
}

Received May 19, 2016; Accepted March 9, 2017

DOI: $10.3892 / \mathrm{ol} .2017 .6414$

\begin{abstract}
There is limited data on the clinical and biological parameters that enable the prediction of the benefits derived from additional chemotherapy after disease progression compared with standard chemotherapy in patients with metastatic colorectal cancer (mCRC). The present study evaluated the role of tumor response as a clinical parameter and single nucleotide polymorphisms (SNPs) as a biomarker to predict the benefit of additional 5-fluorouracil (5-FU) rechallenge chemotherapy in patients with refractory mCRC. Tumor responses were retrospectively reviewed based on the Response Evaluation Criteria in Solid Tumors, early tumor shrinkage (ETS) and depth of response (DoR) following first-line chemotherapy in patients with stage IV CRC. Together with these parameters, SNPs known to be associated with the response to chemotherapy were analyzed with survival outcomes. In total, the tumor responses of 242 patients with mCRC were evaluated. Overall response and ETS were identified in 110 (45.4\%) and 103 patients $(42.6 \%)$, respectively, and the median DoR was $38.5 \pm 30.08 \%$. ETS and DoR were significantly associated with survival outcomes, including progression-free survival, post-progression survival and overall survival. Among these patients, SNPs were analyzed in 171 patients. X-ray repair cross complementing 1 (XRCC1) (AG/AA) with a DoR $>60 \%$, good performance status and the absence of bone lesions were associated with improved overall survival. In patients receiving third-line chemotherapy with 5-FU rechallenge therapy, the methylenetretrahydrofolate reductase (MTHFR) (C677T) CC genotype and a DoR $>60 \%$ were significantly associated with a good prognosis in multivariate analysis.
\end{abstract}

Correspondence to: Dr Sang-Hee Cho, Department of Hemato-Oncology, Chonnam National University Medical School, 42 Jaebong-ro, Gwangju 61186, Republic of Korea

E-mail: shcho@jnu.ac.kr

Key words: colorectal cancer, chemotherapy, early tumor shrinkage, depth of response, polymorphism
$X R C C 1$ (AG/AA) was also associated with a good prognosis in patients with $\mathrm{mCRC}$. Patients with a DoR $>60 \%$ following first-line chemotherapy and a MTHFR (C677T) CC genotype exhibited a survival benefit from 5-FU retreatment. Therefore, the DoR and MTHFR genotype are potential markers for selecting patients with refractory $\mathrm{mCRC}$ that would benefit from 5-FU rechallenge therapy.

\section{Introduction}

The increasing use of palliative chemotherapy with novel targeted agents and improving supportive care during chemotherapy have prolonged the survival and maintained the quality of life of patients with metastatic colorectal cancer (mCRC) $(1,2)$. Based on recent clinical trials (3), the median expected survival time of patients with mCRC is estimated to be $>2$ years. Thus, there is a requirement for techniques to improve the survival of patients with mCRC. Although some patients possess sufficient performance status (PS) to receive further treatment following standard chemotherapy, including oxaliplatin/irinotecan with targeted agents, therapeutic options following standard chemotherapy are lacking. Recently, regorafenib and TAS-102 have been used for third-line chemotherapy $(4,5)$; however, in practice it is difficult to use these drugs due to cost effectiveness. Therefore, physicians often use chemotherapeutic agents that the patient has previously received as a salvage treatment.

Several studies have investigated the reuse of chemotherapeutic drugs, including capecitabine, mitomycin $\mathrm{C}$ and gemcitabine, and have revealed limited effectiveness (6-10). Certain types of chemotherapeutic agents, including S-1 and oxaliplatin combined with 5-fluorouracil (5-FU) or capecitabine, have demonstrated modest efficacy with response rates of $15-20 \%$ (11-14). However, there are no parameters to predict the benefit from rechallenge treatment subsequent to standard chemotherapy.

The optimal methods for determining tumor response have been widely studied previously. The Response Evaluation Criteria in Solid Tumors (RECIST) (15) is the most popular method to assess tumor responses. However, the objective response rate fails to identify strong surrogate markers for 
overall survival (OS) and progression-free survival (PFS), and is also limited in its prediction of OS $(16,17)$. Therefore, early tumor shrinkage (ETS) and depth of response (DoR) have been developed to overcome these problems (16). ETS and DoR have been associated with prolonged PFS, post-progression survival (PPS) and OS $(18,19)$. ETS and DoR were initially developed to predict the efficacy of targeted agents (19), but their roles in conventional chemotherapy have not yet been fully evaluated (20). As a biomarker to predict tumor response, several single-nucleotide polymorphisms (SNPs) have been evaluated as prognostic and predictive markers for the effectiveness of chemotherapy (21-23), although genome-wide association studies are being actively studied in various tumors. The methylenetetrahydrofolate reductase (MTHFR) polymorphism is known to be associated with the metabolism of folate and response of 5-FU treatment in CRC (24-31). Furthermore, the $\mathrm{X}$-ray repair cross-complementing 1 (XRCC1) gene is known to be associated with the response of patients to platinum-based anticancer therapy in CRC (32-36). 5-FU and platinum are basic chemotherapies for MCRC. Thus, such SNPs could be predictive biomarkers of clinical outcome in $\mathrm{mCRC}$.

The present study evaluated tumor response following first-line chemotherapy based on the RECIST, ETS, DoR and SNPs associated with response to chemotherapy, to evaluate their roles as prognostic indicators of survival outcomes. With these results, the SNPs as a biomarker, and ETS and DoR as clinical parameters, were analyzed to define the prognostic significance of 5-FU rechallenge chemotherapy as a third-line of treatment for patients with mCRC.

\section{Materials and methods}

Patients. The data of patients who were diagnosed with mCRC and who underwent chemotherapy at Chonnam National University Hwasun Hospital (Gwangju, Korea) between April 2004 and December 2012 was retrospectively reviewed using medical records and imaging materials. The present study was conducted to evaluate genetic polymorphisms and treatment outcomes in gastrointestinal cancer and was approved by the Institutional Review Board of Chonnam National University Hwasun Hospital. All patients provided written informed consent.

Patients were considered eligible if they had histologically diagnosed adenocarcinoma in the colon or rectum, were initially diagnosed with stage IV (37) or recurrent mCRC, and were suitable for RECIST, ETS and DoR evaluations using target lesion measurements following first-line chemotherapy. In addition, these patients received palliative chemotherapies. Exclusion criteria included no target lesions (based on RECIST), another primary malignancy or severe combined illness that affected chemotherapy treatment. In total, 242 patients were consecutively enrolled from March 2004, and survival data was evaluated until mortality or June 2015. Blood samples from 171/242 patients were available and SNPs known to be associated with chemotherapy response were analyzed in these 171 patients.

Tumor assessment and definition. Tumor assessment by computed tomography (CT) scans were conducted every 8-10 weeks to evaluate disease progression. Undetermined regions were further assessed using magnetic resonance imaging or positron emission tomography CT. Tumor response was assessed according to RECIST (version 1.1) and ETS was defined as an $\geq 20 \%$ decrease in the sum of RECIST target lesions' longest diameters at week 8 compared with the baseline. DoR was defined as the relative change in the sum of the longest diameters of RECIST target lesions at the nadir, in the absence of new lesions or the progression of non-target lesions, compared with the baseline. The outcomes on PFS, PPS and OS were compared. PFS was defined as the time from the beginning of the first-line treatment until documented tumor progression or mortality. OS was defined as the time from the beginning of the first-line treatment until mortality, or the time of the last assessment of disease status. PPS was defined as the time from tumor progression subsequent to first-line treatment until mortality, or the time of the last assessment of disease status. Overall response (OR) was defined as the proportion of patients who achieve a complete or partial response per RECIST criteria. Disease control rate (DCR) was defined as the proportion of patients who achieved a complete response, partial response or stable disease per RECIST criteria.

Genotyping. Genomic DNA was extracted from peripheral blood using a QIAamp DNA Blood Mini kit (Qiagen, Valencia, CA), according to the manufacturer's protocol. Genotyping to analyze polymorphisms was performed using a TaqMan allelic discrimination assay, polymerase chain reaction (PCR)-restriction fragment length polymorphism, high-resolution melting (HRM) analysis and electrophoresis methods. The primer sequences, methods, and references used in the present study are presented in Table I. Genotyping of MTHFR C677T using quantitative PCR was performed with allelic discrimination using dual-labeled probes containing locked nucleic acids (LNA). PCR primers and LNA probes were designed and synthesized by Integrated DNA Technologies (Coralville, IA, USA). Quantitative PCR was performed using a Rotor-Gene 3000 multiplex system (Qiagen) in a 10- $\mu$ l reaction volume containing $200 \mathrm{nM}$ PCR primer, $10 \mathrm{nM}$ of each probe, $0.5 \mathrm{U}$ f-taq polymerase (Solgent, Daejeon, Korea) and $40 \mathrm{ng}$ of genomic DNA. The thermocycling conditions were as follows: Initial 5 -min hold at $95^{\circ} \mathrm{C}$; followed by 40 cycles at $95^{\circ} \mathrm{C}$ for $5 \mathrm{sec}$ and $64^{\circ} \mathrm{C}$ for $30 \mathrm{sec}$. HRM genotyping for glutathione S-transferase pi 1 (GSTP1) rs1695, XRCC1 rs25487 and excision repair 1 endonuclease non-catalytic subunit (ERCC1) rs11615 was performed in $10-\mu l$ reaction volumes with $200 \mathrm{nM}$ PCR primer, $1 \mu$ MSyto 9 fluorescent dye, $0.5 \mathrm{U}$ f-Taq polymerase, and $40 \mathrm{ng}$ of genomic DNA, using a Rotor-Gene 6000 high-resolution system (Qiagen). Genotyping for 28-bp thymidylate synthetase variable number of tandem repeat $(2 R \rightarrow 3 R)$ (TS VNTR) genotypes in the 5'untranslated region of the TS gene was conducted using a protocol described by Horie et al (21). The TSSNP, rs34743033, involving the 12th nucleotide of the second repeat of the $3 \mathrm{R}$ alleles in TS VNTR was genotyped by digesting the TS VNTR PCR products with HaeIII (Takara Bio, Inc., Otsu, Japan) followed by electrophoresis on a $6 \%$ polyacrylamide gel and visualization with ethidium bromide. TSSNP rs34743033 genotypes of the patients were classified in two groups: High expression type $(2 \mathrm{R} 3 \mathrm{G}, 3 \mathrm{C} 3 \mathrm{G}$ and $3 \mathrm{G} 3 \mathrm{G})$ and low expression type $(2 \mathrm{R} 2 \mathrm{R}$, $2 \mathrm{R} / 3 \mathrm{C}$ and $3 \mathrm{C} 3 \mathrm{C})(38)$. 
Table I. Sequences of primers and method used by genotype.

\begin{tabular}{|c|c|c|c|c|}
\hline Gene and rs number & Genotype & Primers and probe & Method & (Refs.) \\
\hline MTHFR677 rs 1801133 & C/T, Ala222 Val & $\begin{array}{l}\text { F, 5'-CTTTGAGGCTGACCTGAAGC-3' } \\
\text { R, 5'-TCACAAAGCGGAAGAA TGTG-3' } \\
\text { aLNA probes for C allele : 5'-FAM-ATG GcT } \\
\text { ccc-BHQ1-3' } \\
\text { LNA probes for T allele: 5'-cy5-cgA CTc cCg } \\
\text { C-BHQ2-3' }\end{array}$ & TaqMan RQ & (46) \\
\hline GSTP1 rs1695 & A/G, Val105Ile & $\begin{array}{l}\text { F, 5'-TGGTGGACATGGTGAATGAC-3' } \\
\text { R, 5'-TGCAGATGCTCACATAGTTGG-3' }\end{array}$ & HRM & $(47)$ \\
\hline XRCC1 rs 25487 & G/A, Arg399Gln & $\begin{array}{l}\text { F, 5'-TAAGGAGTGGGTGCTGGACT-3' } \\
\text { R, 5'-ATTGCCCAGCACAGGATAAG-3' }\end{array}$ & HRM & Present \\
\hline ERCC1 rs 11615 & C/T, Asn118Asn & $\begin{array}{l}\text { F, 5'-TCCCTATTGATGGCTTCTGC-3' } \\
\text { F, 5'-GAGCTCACCTGAGGAACAGG-3' }\end{array}$ & HRM & Present \\
\hline$T S 2 R 3 R$ & $V N T R$ & $\begin{array}{l}\text { F, 5'-GTG GCT CCT GCG TTT CCC CC-3' } \\
\text { R, 5'-CCA AGC TTG GCT CCG AGC } \\
\text { CGG CCA CAG GCA TGG CGC GG-3' }\end{array}$ & PCR, EP & (21) \\
\hline TSrs34743033 & $3 R / G / C$ & & RFLP:HaeIII,EP & \\
\hline
\end{tabular}

Statistical analysis. Continuous variables were expressed as the mean \pm standard deviation. $\chi^{2}$ or Fisher's exact tests were used to compare categorical variables, and Student's t-test was used to compare continuous variables.

ETS, DoR and RECIST responses were examined for their prognostic impact on PFS, PPS and OS. DoR was evaluated as five levels based on quintile distribution with increments of $20 \%$. The cut-off value of DoR $>60 \%$ was achieved through this categorizing. The Kaplan-Meier estimator method was used to examine cumulative survival and time to mortality or progression, and the log-rank test was used to compare differences between groups. The factors identified as significant by univariate analysis were subjected to stepwise multivariate analysis (forward selection) to determine which factors retained statistical significance, and which were merely dependent on other factors. Multivariate analyses were performed using the Cox proportional hazards model and logistic regression analysis to identify independent prognostic variables. $\mathrm{P}<0.05$ (two-tailed) was considered to indicate a statistically significant difference. All analyses were performed using SPSS software (version 21.0; IBM SPSS, Armonk, NY, USA).

\section{Results}

Baseline characteristics and tumor responses following first-line chemotherapy. The baseline patient characteristics and types of first-line chemotherapies are illustrated in Table II. The median patient age was 67 years, and 139 (57.4\%) patients were $>65$ years of age. Of the patients in the study, $42(17.4 \%)$ patients had previously received adjuvant chemotherapy prior to recurrence, and 14 (5.8\%) patients received a target agent combined with systemic chemotherapy. First-line chemotherapy regimens included FOLFOX (oxaliplatin with 5-FU; 51.7\%), FOLFIRI (irinotecan with 5-FU; 34.7\%) and capecitabine (13.6\%). In total, $187(76.9 \%)$ patients received second-line chemotherapy and 119 (49.2\%) patients received third-line chemotherapy (data not shown).

Based on RECIST, the OR following first-line chemotherapy was $41.3 \%(n=100)$ (data not shown). The median percentage of tumor shrinkage at week 8 was $26.47 \pm 24.94 \%$, and the percentage of ETS was $42.6 \%(n=103)$ in patients without progression. The median DoR was $38.53 \pm 30.08 \%$ in patients without progression. The median OS, PPS and PFS of all enrolled patients were $18.00 \pm 18.09$ [95\% confidence interval (CI), 20.13-24.66], 11.13 \pm 14.78 (95\% CI, 12.93-16.63) and $5.95 \pm 7.12$ (95\% CI, 6.74-8.58) months, respectively (data not shown).

Association between tumor response and survival parameters. An OR was associated with a significantly longer PFS (9.7 vs. 3.1 months; $\mathrm{P}<0.001)$ and $\mathrm{OS}(23.7$ vs. 16.9 months; $\mathrm{P}=0.008)$ compared with stable or progressive disease. However, there was no association between OR and PPS (12.8 vs. 11.7 months; $\mathrm{P}=0.364$ ) (data not shown).

Patients who achieved ETS had a significantly longer PFS compared with non-ETS-achieving patients (9.1 vs. 3.1 months; $\mathrm{P}<0.001)$, PPS (13.7 vs. 11.2 months; $\mathrm{P}=0.044)$, and $\mathrm{OS}$ (23.7 vs. 16.8 months; $\mathrm{P}<0.001$ ) (Fig. 1). To determine the association between DoR and survival, patients were randomly divided into five groups (I-V). The median OS of each DoR group was 19.6, 18.1, 21.8, 25.5 and 36.1 months in groups $\mathrm{I}-\mathrm{V}$, respectively $(\mathrm{P}=0.006)$. In addition, PFS $(5.77,7.43,9.07$, 
Table II. Baseline patient characteristics according to the number of chemotherapy treatments $(n=242)$.

\begin{tabular}{|c|c|}
\hline Characteristic & Number of patient \\
\hline \multicolumn{2}{|l|}{ Age (years) } \\
\hline$<65$ & $103(42.6)$ \\
\hline$>65$ & $139(57.4)$ \\
\hline \multicolumn{2}{|l|}{ Sex } \\
\hline Male & $151(62.4)$ \\
\hline Female & $91(37.6)$ \\
\hline \multicolumn{2}{|l|}{ ECOG PS } \\
\hline 0 & $88(36.4)$ \\
\hline 1 & $119(49.2)$ \\
\hline 2 & $30(12.4)$ \\
\hline 3 & $5(2.0)$ \\
\hline \multicolumn{2}{|l|}{ Primary tumor site } \\
\hline Colon & $195(80.6)$ \\
\hline Rectum & $47(19.4)$ \\
\hline \multicolumn{2}{|l|}{ Tumor grade } \\
\hline 1 & $67(27.7)$ \\
\hline 2 & $126(52.1)$ \\
\hline 3 & 28 (11.6) \\
\hline NA & $21(8.6)$ \\
\hline \multicolumn{2}{|l|}{ Stage at diagnosis } \\
\hline Metastatic & $166(68.6)$ \\
\hline Recurred & $68(28.1)$ \\
\hline NA & $8(3.3)$ \\
\hline \multicolumn{2}{|l|}{ Metastatic site } \\
\hline Liver & $157(64.9)$ \\
\hline Lung & $75(31.0)$ \\
\hline Bone & $10(4.1)$ \\
\hline LN & $48(19.8)$ \\
\hline $\begin{array}{l}\text { Previous adjuvant } \\
\text { chemotherapy }\end{array}$ & $42(17.4)$ \\
\hline Use of target agent & $14(5.8)$ \\
\hline \multicolumn{2}{|l|}{ First-line regimen } \\
\hline 5-FU+oxaliplatin & $125(51.7)$ \\
\hline 5-FU+irinotecan & $84(34.7)$ \\
\hline Capecitabine & $33(13.6)$ \\
\hline
\end{tabular}

NA, not accessible; 5-FU, 5-fluorouracil; ECOG PS, Eastern Cooperative Oncology Group Performance Status; LN, lymph node.

10.00 and 13.53 months in groups $\mathrm{I}-\mathrm{V}$, respectively; $\mathrm{P}=0.001$ ) and PPS (11.20, 8.50, 12.17, 17.67 and 19.17 months in group $\mathrm{I}-\mathrm{V}$, respectively; $\mathrm{P}=0.012$ ) were also significantly associated with DoR (Fig. 2). Patients with a DoR $\geq 60 \%$ had significantly improved PFS (11.6 vs. 4.8 months; $\mathrm{P}<0.001)$, PPS (18.4 vs. 10.1 months; $\mathrm{P}<0.001)$ and $\mathrm{OS}(31.6$ vs. 17.2 months; $\mathrm{P}<0.001)$ compared with patients with a DoR $<60 \%$.

Association between genotyping of SNPs and survival parameters. Of the patients in the current study, 171 were genotyped for SNPs known to be associated with chemotherapy responses. Genotype and haplotype distributions of the patients are illustrated in Table III. According to univariate analyses, the GSTP1 (Ile105 Val) AA genotype, lymph node metastasis, liver metastasis, OR, ETS and a DoR $\geq 60 \%$ were significantly associated with PFS. Using multivariate analyses, GSTP1 (Ile105Val) AA, a DoR $\geq 60 \%$ and OR were independent prognostic factors for an improved PFS, and liver metastasis for a worse PFS (Table IV). The XRCC1 (G399A) AA/AG genotype, ECOG PS 0-1, stage IV cancer at diagnosis, bone metastasis, ETS and a DoR $\geq 60 \%$ were associated with improved OS using univariate analyses. The XRCC1 (G399A) AA/AG genotype, ECOG PS $0-1$, a DoR $\geq 60 \%$ and the absence of bone metastasis were independent factors associated with an improved OS.

Analysis of tumor response and SNPs as prognostic markers for benefiting from third-line chemotherapy. Amongst the 171 patients whose SNPs were analyzed, 88 received third-line chemotherapy following the second progression. Third-line chemotherapy included therapy using 5-FU $[\mathrm{n}=63,71.6 \%$; capecitabine $(n=47)$; S-1 $(n=8) ; 5-F U$ with leucovorin $(n=8)]$, 5-FU with oxaliplatin $(n=18,20.5 \%), 5-F U$ with irinotecan $(n=6,6.8 \%)$ and 5-FU with other drugs $(n=1,1.1 \%)$ (data not shown). Patients receiving third-line chemotherapy were younger compared with patients not receiving third-line chemotherapy (median age 62.5 vs. 68.0 years, respectively; $\mathrm{P}=0.023)$. There was no significant difference in PS between the two groups (PS $0-187.5 \%$ vs. 76.8\%; $\mathrm{P}=0.074$ ). The response rate was $17.0 \%(n=15)$ and the disease control rate was $45.5 \%(n=40)$. However, the number of patients in each treatment group was small and the efficacies according to the agents used for treatment were not evaluated. The response duration from the start of chemotherapy to progression was 18.7 \pm 19.0 weeks (data not shown).

To identify patients that benefited from additional chemotherapy subsequent to the second progression, patients were grouped according to the average OS of all patients ( $\geq$ or $<23$ months). The longer OS group included 50 patients, and the shorter OS group included 38 patients. No significantly different clinical characteristics between the longer and shorter OS groups were identified (data not shown).

Univariate analyses revealed that a XRCC1 (G399A) AA/AG genotype, ETS, a MTHFR (C677T) CC genotype and a DoR $\geq 60 \%$ were frequently present in the longer OS group. However, multivariate analyses demonstrated that a MTHFR (C677T) CC genotype [hazard ratio (HR), 2.755; 95\% CI, 1.057-7.143; $\mathrm{P}=0.038]$ and $\mathrm{DoR} \geq 60 \%$ (HR, 6.469; 95\% $\mathrm{CI}=1.701-24.594 ; \mathrm{P}=0.006)$ were significant prognostic factors for improved OS following third-line chemotherapy (Table V).

\section{Discussion}

The present analysis is the first attempt, to the best of our knowledge, to evaluate parameters indicating benefit from 5-FU retreatment as a third-line chemotherapy for mCRC. The data of 243 patients with $\mathrm{mCRC}$ was retrospectively analyzed in the present study. Due to the fact that targeted treatments were not adopted and reimbursed at this time in South Korea, 

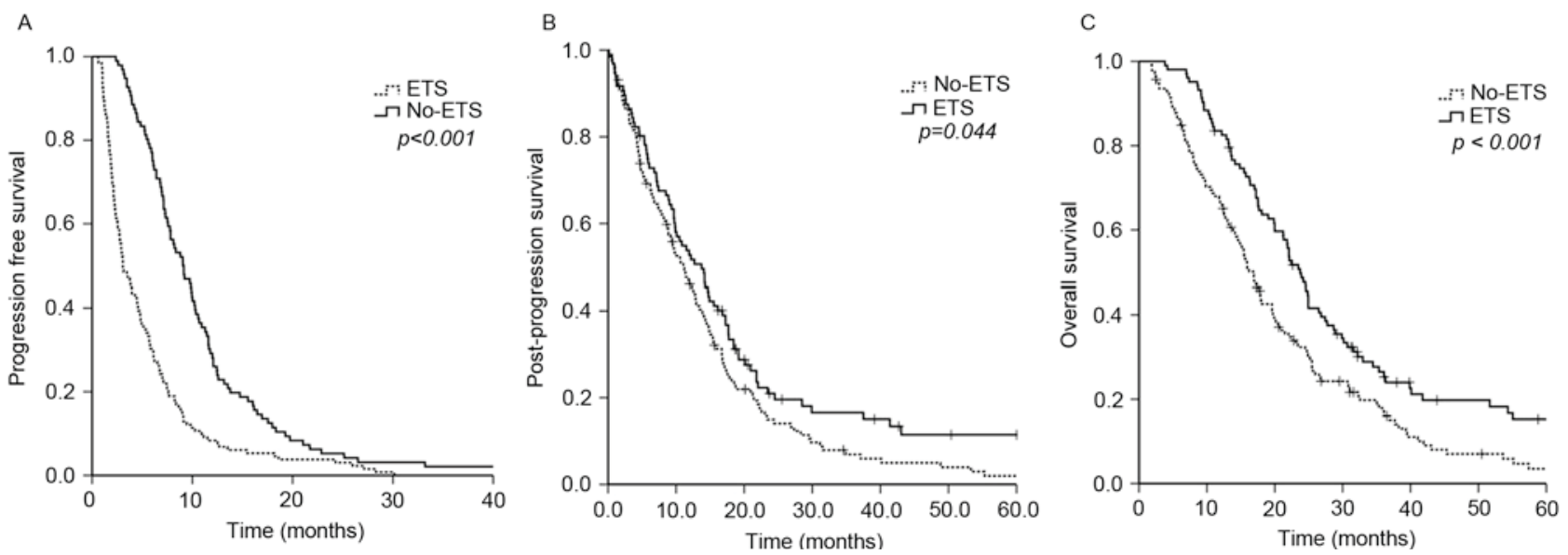

Figure 1. Survival outcomes according to ETS. (A) progression-free survival, (B) post-progression survival and (C) overall survival. ETS, early tumor response.

A

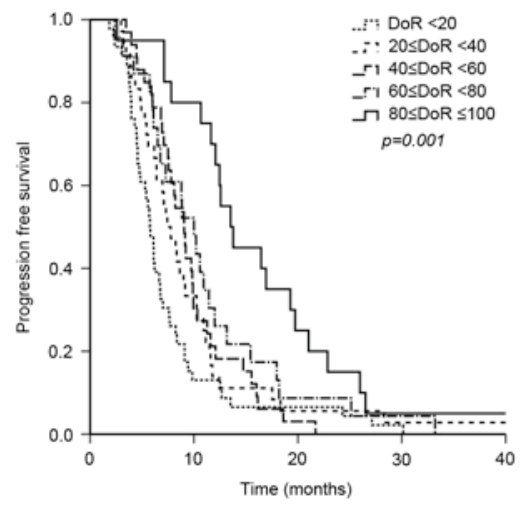

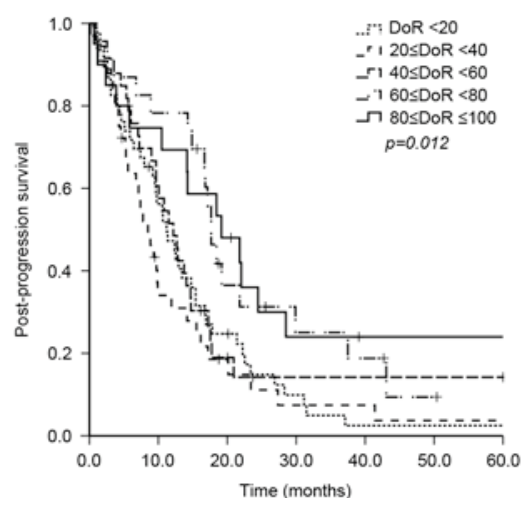

c

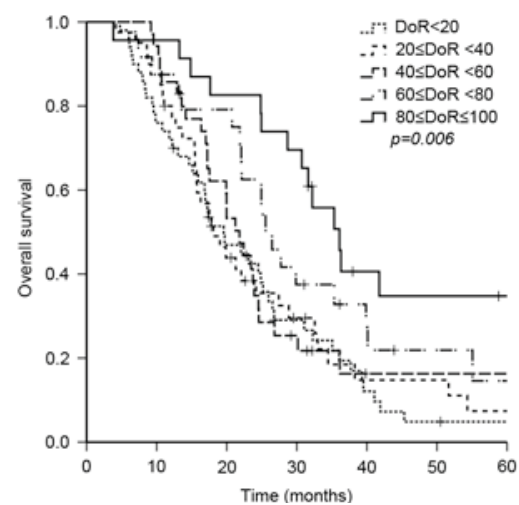

Figure 2. Survival outcomes according to DoR. (A) progression-free survival, (B) post-progression survival and (C) overall survival. DoR, depth of response.

the majority of patients included in the present study received conventional chemotherapies. Therefore, the median OS in the enrolled patients was only 18 months. As discussed above, there are a number of treatment options for refractory $\mathrm{mCRC}$, and rechallenge chemotherapy may be used as salvage chemotherapy. In the current study, 36\% $(n=88)$ patients received third-line chemotherapy, including 5-FU following the second progression. Retreatment with 5 -FU resulted in an OR of $17.0 \%$ and a DCR of $45.5 \%$ following 18 weeks of response. These results are slightly improved compared with those of previous studies $(9-11,13,22)$.

Although there is a lack of evidence to determine what molecular mechanisms underlie the effects of rechallenge treatments, a recent clinical result suggests a rationale for this treatment approach $(19,23)$. In the updated results of the Triplet Plus Bevacizumab study, 5-FU, oxaliplatin and irinotecan [FOLFOXIRI] with bevacizumab as a first-line treatment revealed promising efficacy compared with FOLFIRI with bevacizumab (23). Notably, in the present study of FOLFOXIRI with bevacizumab as a first-line treatment was effective and improved PPS to $>17$ months. Taking into account the fact that patients had already been exposed to four agents (FOLFOXIRI with bevacizumab) and that $80 \%$ of the patients received second-line chemotherapy, including previous chemotherapy with or without targeted agents, subsequent rechallenge treatment may be beneficial to prolong survival.

Tyrosine kinase inhibitors for patients with lung cancer exhibiting mild and asymptomatic progression were continued subsequent to initial evidence of progressive disease in previous studies $(20,39)$. Radiological progression does not always involve all tumor sites possessing the same cause of resistance; certain tumor sites could be sensitive to previously used chemotherapy at the time of RECIST progression. These results suggest that rechallenge or retreatment with previously used agents could prolong the survival of patients with mCRC. However, further studies are required to verify the effectiveness of retreatment with 5-FU as a third-line chemotherapy for the treatment of patients with mCRC.

When considering the lack of current indicators for retreatment benefit, the results from the current study could aid in identifying patients who may benefit from 5-FU rechallenge chemotherapy on the basis of tumor response and tumor sensitivity. Multivariate analyses in the present study revealed that the MTHFR (C677T) CC genotype and a DoR $\geq 60 \%$ were associated with improved OS following receiving third-line treatment using 5-FU, regardless of age or PS score.

The reduced activity of MTHFR in allele $T$ carriers increases the availability of 5,10-methylenetetrahydrofolate 
Table III. Genotype and haplotype distribution of single-nucleotide polymorphisms $(\mathrm{n}=171)$.

\begin{tabular}{lc}
\hline Genotype & Number of patients (\%) \\
\hline MTHFR (C677T) & $66(38.8)$ \\
CC & $164(61.2)$ \\
CT, TT & \\
XRCC1 (G399A) & $98(57.6)$ \\
GG & $72(42.4)$ \\
AA, AG & \\
GSTP1 (Ile105Val) & $81(47.6)$ \\
AA & $89(52.4)$ \\
AG, GG & \\
ERCC1 (T19007C) & $89(52.4)$ \\
CC & $81(47.6)$ \\
TT, TC & \\
TS2R3R & $117(68.8)$ \\
3R/3R & $53(31.2)$ \\
2R/2R, 2R/3R & \\
TSrs34743033 & $122(71.8)$ \\
High expression & $48(28.2)$ \\
Low expression & \\
\hline
\end{tabular}

${ }^{a}$ Low expression alleles (2R/2R, 2R/3C AND 3C/3C) and high expression alleles $(2 \mathrm{R} / 3 \mathrm{G}, 3 \mathrm{C} / 3 \mathrm{G}$, and $3 \mathrm{G} / 3 \mathrm{G})$. MTHFR, methylenetretrahydrofolate reductase; $\mathrm{XRCC1}$, X-ray repair cross complementing 1; GSTP1, glutathione S-transferase pi 1; TS, thymidylate synthetase.

Table IV. Multivariate analysis of the association between genotyping of single-nucleotide polymorphisms and survival parameters $(n=171)$.

\begin{tabular}{lccc}
\hline Variable & HR & $95 \%$ CI & P-value \\
\hline PFS & & & \\
Liver metastasis & 0.549 & $0.384-0.784$ & 0.001 \\
GSTP1 (Ile105Val) AA & 1.390 & $1.007-1.918$ & 0.045 \\
DoR $\geq 60 \%$ & 1.639 & $1.074-2.500$ & 0.022 \\
OR & 2.424 & $1.705-3.445$ & $<0.0001$ \\
OS & & & \\
Bone metastasis & 0.310 & $0.147-0.655$ & 0.002 \\
XRCC1 (G399A) AA, AG & 1.681 & $1.190-2.375$ & 0.003 \\
ECOG PS 0-1 & 1.862 & $1.212-2.857$ & 0.005 \\
DoR $\geq 60 \%$ & 2.195 & $1.443-3.341$ & $<0.0001$ \\
\end{tabular}

OR, overall response; DoR, depth of response; ECOG PS, Eastern Cooperative Oncology Group Performance Status; PFS, progression-free survival; OS, overall survival; HR, hazard ratio; CI, confidence interval; XRCC1, X-ray repair cross complementing 1.

(5,10-MTHF), a necessary cofactor for 5-FU inhibition of TYMS. Using an in vitro model, 5-FU demonstrated increased activity in the presence of the MTHFR $677 T$ allele in CRC (40).
Table V. Univariate and multivariate analyses of the benefit of third-line chemotherapy $(\mathrm{n}=88)$.

\begin{tabular}{lccc}
\hline Variable & HR & $95 \%$ CI & P-value \\
\hline Univariate analysis & & & \\
XRCC1 (G399A) AA, AG & 2.571 & $1.079-6.130$ & 0.033 \\
ETS & 2.659 & $1.088-6.501$ & 0.032 \\
MTHFR (C677T) CC & 2.801 & $1.126-6.944$ & 0.027 \\
DoR $\geq 60 \%$ & 6.562 & $1.766-24.392$ & 0.005 \\
Multivariate analysis & & & \\
$\quad$ MTHFR (C677T) CC & 2.755 & $1.057-7.143$ & 0.038 \\
DoR $\geq 60 \%$ & 6.469 & $1.701-24.594$ & 0.006 \\
\hline
\end{tabular}

HR, hazard ratio; CI, confidence interval; ETS, early tumor response; DoR, depth of response; $M T H F R$, methylenetretrahydrofolate reductase; XRCC1, X-ray repair cross complementing 1.

However, in vivo studies here revealed conflicting results. Several studies demonstrated that the $677 T$ allele had a positive or no effect on survival (24-27), while other studies revealed a negative effect on the treatment response $(30,31)$. The present study revealed that the MTHFR (C677T) CC genotype was associated with improved OS during additional 5-FU chemotherapy. Possible explanations for these conflicting results may involve the cellular availability of 5,10-MTHF, which depends on the MTHFR genotype and other factors, including dietary folate $(31,39,41)$.

The association between SNPs and tumor responses have been evaluated in a number of previous studies. XRCCI repairs single-strand DNA breaks by encoding a protein that defends breaks (32), and the XRCC1 protein is important for repairing the DNA damage induced by platinum-based anticancer drugs (42). Several studies have reported the efficacy of platinum-based anticancer therapy as a predictive marker in CRC (32-36). Similar to previous investigations, the current study also demonstrated good prognosis in terms of OS in patients with the XRCCl (G399A) AA/AG genotype; however, this genotype was not associated with an improved PFS.

Although the individual development and survival mechanisms of cancer cells have been studied, human cancer exhibits intratumoral heterogeneity in phenotypic features. These include cellular morphology, gene expression, metabolism, motility, and angiogenic, proliferative, immunogenic and metastatic potential (43). Opposed to SNPs as markers for innate tumor sensitivity, ETS and DoR could be surrogate markers covering various responses according to heterogeneous tumor characteristics, as they measure changes to the size of the tumor mass following chemotherapy.

ETS and DoR have been demonstrated to be associated with OS and PFS in several previous studies $(18,19,44,45)$. In the present study, the majority of patients were treated with conventional chemotherapy to evaluate ETS and DoR as prognostic markers when using targeted agents, unlike previous studies. Although the current study was retrospective, it suggested that ETS and DoR can be used as prognostic factors for survival outcomes, including PFS, PPS and OS, in patients with mCRC who receive conventional cytotoxic chemotherapy. 
The cutoff value of DoR $\geq 60 \%$ was achieved by categorizing the patients into five DoR groups based on increments of $20 \%$, with similar results to a previous study showing an optimal DoR of $62.4 \%$ (18).

In conclusion, the present study demonstrated that DoR and $X R C C l$ (AG/AA) are associated with more improved prognoses in patients with refractory $\mathrm{mCRC}$ receiving palliative chemotherapy. In particular, patients with a DoR $>60 \%$ following first-line chemotherapy and an MTHFR (C677T) CC genotype exhibited a survival benefit from 5-FU retreatment as a form of third-line chemotherapy. Therefore, DoR and MTHFR genotype are potential clinical markers for selecting patients with refractory $\mathrm{mCRC}$ that would benefit from 5-FU rechallenge therapy.

\section{Acknowledgements}

The English in this document has been checked by at least two professional editors, both native speakers of English. For a certificate, please see http://www.textcheck.com/ certificate/BuYyzv.

\section{References}

1. Van Cutsem E, Cervantes A, Nordlinger B and Arnold D; ESMO Guidelines Working Group: Metastatic colorectal cancer: ESMO clinical practice guidelines for diagnosis, treatment and follow-up. Ann Oncol 25 (Suppl 3): iii1-iii9, 2014.

2. Benson AB III, Venook AP, Bekaii-Saab T, Chan E, Chen YJ, Cooper HS, Engstrom PF, Enzinger PC, Fenton MJ, Fuchs CS, et al: Colon cancer, version 3.2014. J Natl Compr Canc Netw 12: 1028-1059, 2014.

3. Van Cutsem E, Köhne CH, Láng I, Folprecht G, Nowacki MP, Cascinu S, Shchepotin I, Maurel J, Cunningham D, Tejpar S, et al: Cetuximab plus irinotecan, fluorouracil, and leucovorin as first-line treatment for metastatic colorectal cancer: Updated analysis of overall survival according to tumor KRAS and BRAF mutation status. J Clin Oncol 29: 2011-2019, 2011.

4. Grothey A, Van Cutsem E, Sobrero A, Siena S, Falcone A, Ychou M, Humblet Y, Bouché O, Mineur L, Barone C, et al: Regorafenib monotherapy for previously treated metastatic colorectal cancer (CORRECT): An international, multicentre, randomised, placebo-controlled, phase 3 trial. Lancet 381: 303-312, 2013.

5. Mayer RJ, Van Cutsem E, Falcone A, Yoshino T, Garcia-Carbonero R, Mizunuma N, Yamazaki K, Shimada Y, Tabernero J, Komatsu Y, et al: Randomized trial of TAS-102 for refractory metastatic colorectal cancer. N Engl J Med 372: 1909-1919, 2015.

6. Nielsen DL, Palshof JA, Larsen FO, Jensen BV and Pfeiffer P: A systematic review of salvage therapy to patients with metastatic colorectal cancer previously treated with fluorouracil, oxaliplatin and irinotecan +/- targeted therapy. Cancer Treat Rev 40: 701-715, 2014.

7. Scartozzi M, Falcone A, Pucci F, Braconi C, Pierantoni C, Cavanna L, Franciosi V, Berardi R, Beretta G, Masi G, et al: Capecitabine and mitomycin $\mathrm{C}$ may be an effective treatment option for third-line chemotherapy in advanced colorectal cancer. Tumori 92: 384-388, 2006.

8. Vormittag L, Kornek GV, Gruhsmann B, Lenauer A, Föger A Depisch D, Lang F and Scheithauer W: UFT/leucovorin and mitomycin $\mathrm{C}$ as salvage treatment in patients with advanced colorectal cancer-a retrospective analysis. Anticancer Drugs 18: 709-712, 2007

9. Gubanski M, Naucler G, Almerud A, Lideståhl A and Lind PA: Capecitabine as third line therapy in patients with advanced colorectal cancer. Acta Oncol 44: 236-239, 2005.

10. Lee S, Kwon HC, Kim SH, OH YS, Lee HJ, Yoon HH, Choi JH and Kim JH: Capecitabine monotherapy, and the clinical significance of neutrophil-lymphocyte ratio versus platelet-lymphocyte ratio in patients with metastatic colorectal cancer. In: ASCO Annual Meeting Proceedings, p660, 2012.
11. Jeung HC, Rha SY, Cho BC, Yoo NC, Roh JK, Roh WJ, Chung HC and Ahn JB: A phase II trial of S-1 monotherapy in metastatic colorectal cancer after failure of irinotecan- and oxaliplatin-containing regimens. Br J Cancer 95: 1637-1641, 2006.

12. Sun Jin Sym, Junshik Hong, Hee Kyung Ahn, Jinny Park, Eun Kyung Cho, Jae Hoon Lee, Won-Suk Lee, Jeong-Heum Baek, Yeon Ho Park, Dong Bok Shin, et al: A phase II trial of salvage treatment with gemcitabine and S-1 combination in heavily pretreated patients with metastatic colorectal cancer. J Clin Oncol 31 (Suppl 4): S488, 2013.

13. Santini D, Vincenzi B, La Cesa A, Caricato M, Schiavon G, Spalletta B, Di Seri M, Coppola R, Rocci L and Tonini G: Continuous infusion of oxaliplatin plus chronomodulated capecitabine in 5-fluorouracil- and irinotecan-resistant advanced colorectal cancer patients. Oncology 69: 27-34, 2005.

14. Townsend AR, Bishnoi S, Broadbridge V, Beeke C, Karapetis CS, Jain K, Luke C, Padbury R and Price TJ: Rechallenge with oxaliplatin and fluoropyrimidine for metastatic colorectal carcinoma after prior therapy. Am J Clin Oncol 36: 49-52, 2013.

15. Eisenhauer EA, Therasse P, Bogaerts J, Schwartz LH, Sargent D, Ford R, Dancey J, Arbuck S, Gwyther S, Mooney M, et al: New response evaluation criteria in solid tumours: Revised RECIST guideline (version 1.1). Eur J Cancer 45: 228-247, 2009.

16. Grothey A, Hedrick EE, Mass RD, Sarkar S, Suzuki S, Ramanathan RK, Hurwitz HI, Goldberg RM and Sargent DJ: Response-independent survival benefit in metastatic colorectal cancer: A comparative analysis of N9741 and AVF2107. J Clin Oncol 26: 183-189, 2008.

17. Buyse M, Thirion P, Carlson RW, Burzykowski T, Molenberghs G and Piedbois P: Relation between tumour response to first-line chemotherapy and survival in advanced colorectal cancer: A meta-analysis. Meta-analysis group in cancer. Lancet 356: 373-378, 2000

18. Piessevaux H, Buyse M, Schlichting M, Van Cutsem E, Bokemeyer C, Heeger S and Tejpar S: Use of early tumor shrinkage to predict long-term outcome in metastatic colorectal cancer treated with cetuximab. J Clin Oncol 31: 3764-3775, 2013.

19. Cremolini C, Loupakis F, Antoniotti C, Lonardi S, Masi G, Salvatore L, Cortesi E, Tomasello G, Spadi R, Zaniboni A, et al: Early tumor shrinkage and depth of response predict long-term outcome in metastatic colorectal cancer patients treated with first-line chemotherapy plus bevacizumab: Results from phase III TRIBE trial by the Gruppo Oncologico del Nord Ovest. Ann Oncol 26: 1188-1194, 2015.

20. Ettinger DS, Wood DE, Akerley W, Bazhenova LA, Borghaei H, Camidge DR, Cheney RT, Chirieac LR, D'Amico TA, Demmy TL, et al: Non-small cell lung cancer, version 6.2015 . J Natl Compr Canc Netw 13: 515-524, 2015.

21. Horie N, Aiba H, Oguro K, Hojo H and Takeishi K: Functional analysis and DNA polymorphism of the tandemly repeated sequences in the 5'-terminal regulatory region of the human gene for thymidylate synthase. Cell Struct Funct 20: 191-197, 1995.

22. Lee DJ, Lee J, Lee HY, Lim T, Lee SJ, Yi SY, Park SH, Park JO, Lim HY, Kang WK and Park YS: Salvage S-1 monotherapy in metastatic colorectal cancer patients who failed irinotecan-based or oxaliplatin-based chemotherapy. Med Oncol 28 (Suppl 1): S291-S294, 2011

23. Cremolini C,Loupakis F, Antoniotti C,Lupi C, Sensi E, Lonardi S, Mezi S, Tomasello G, Ronzoni M, Zaniboni A, et al: FOLFOXIRI plus bevacizumab versus FOLFIRI plus bevacizumab as first-line treatment of patients with metastatic colorectal cancer: Updated overall survival and molecular subgroup analyses of the open-label, phase 3 TRIBE study. Lancet Oncol 16: 1306-1315, 2015.

24. Etienne-Grimaldi MC, Milano G, Maindrault-Goebel F, Chibaudel B, Formento JL, Francoual M, Lledo G, André T, Mabro M, Mineur L, et al: Methylenetetrahydrofolate reductase (MTHFR) gene polymorphisms and FOLFOX response in colorectal cancer patients. Br J Clin Pharmacol 69: 58-66, 2010

25. Jakobsen A, Nielsen JN, Gyldenkerne N and Lindeberg J: Thymidylate synthase and methylenetetrahydrofolate reductase gene polymorphism in normal tissue as predictors of fluorouracil sensitivity. J Clin Oncol 23: 1365-1369, 2005.

26. Cohen V, Panet-Raymond V, Sabbaghian N, Morin I, Batist G and Rozen R: Methylenetetrahydrofolate reductase polymorphism in advanced colorectal cancer: A novel genomic predictor of clinical response to fluoropyrimidine-based chemotherapy. Clin Cancer Res 9: 1611-1615, 2003. 
27. Castillo-Fernández O, Santibáñez M, Bauza A, Calderillo G, Castro C, Herrera R, Serrano A, Arrieta O and Herrera LA: Methylenetetrahydrofolate reductase polymorphism $(677 \mathrm{C}>\mathrm{T})$ predicts long time to progression in metastatic colon cancer treated with 5-fluorouracil and folinic acid. Arch Med Res 41: 430-435, 2010.

28. Suh KW, Kim JH, Kim DY, Kim YB, Lee C and Choi S: Which gene is a dominant predictor of response during FOLFOX chemotherapy for the treatment of metastatic colorectal cancer, the MTHFR or XRCC1 gene? Ann Surg Oncol 13: 1379-1385, 2006.

29. Marcuello E, Altés A, Menoyo A, Rio ED and Baiget M: Methylenetetrahydrofolate reductase gene polymorphisms: Genomic predictors of clinical response to fluoropyrimidine-based chemotherapy? Cancer Chemother Pharmacol 57: 835-840, 2006.

30. Derwinger K, Wettergren $Y$, Odin E, Carlsson G and Gustavsson B: A study of the MTHFR gene polymorphism C677T in colorectal cancer. Clin Colorectal Cancer 8: 43-48, 2009.

31. Sharma R, Hoskins JM, Rivory LP, Zucknick M, London R, Liddle $\mathrm{C}$ and Clarke SJ: Thymidylate synthase and methylenetetrahydrofolate reductase gene polymorphisms and toxicity to capecitabine in advanced colorectal cancer patients. Clin Cancer Res 14: 817-825, 2008.

32. Wu H, Xu C, Chen G and Wang J: X-ray repair cross-complementing 1 polymorphism and prognosis of platinum-based chemotherapy in gastric and colorectal cancer: A meta-analysis. J Gastroenterol Hepatol 29: 926-933, 2014.

33. Liang J, Jiang T, Yao RY, Liu ZM, Lv HY and Qi WW: The combination of ERCC1 and XRCC1 gene polymorphisms better predicts clinical outcome to oxaliplatin-based chemotherapy in metastatic colorectal cancer. Cancer Chemother Pharmacol 66 : 493-500, 2010.

34. Ye F, Liu Z, Tan A, Liao M, Mo Z and Yang X: XRCC1 and GSTP1 polymorphisms and prognosis of oxaliplatin-based chemotherapy in colorectal cancer: A meta-analysis. Cancer Chemother Pharmacol 71: 733-740, 2013.

35. Lv H, Li Q, Qiu W, Xiang J, Wei H, Liang H, Sui A and Liang J: Genetic polymorphism of XRCC1 correlated with response to oxaliplatin-based chemotherapy in advanced colorectal cancer. Pathol Oncol Res 18: 1009-1014, 2012.

36. Huang MY, Huang ML, Chen MJ, Lu CY, Chen CF, Tsai PC, Chuang SC, Hou MF, Lin SR and Wang JY: Multiple genetic polymorphisms in the prediction of clinical outcome of metastatic colorectal cancer patients treated with first-line FOLFOX-4 chemotherapy. Pharmacogenet Genomics 21: 18-25, 2011.
37. Edge SB and Compton CC: The American Joint Committee on Cancer: The 7th edition of the AJCC cancer staging manual and the future of TNM. Ann Surg Oncol 17: 1471-1474, 2010.

38. Kawakami K and Watanabe G: Identification and functional analysis of single nucleotide polymorphism in the tandem repeat sequence of thymidylate synthase gene. Cancer Res 63: 6004-6007, 2003.

39. Ruzzo A, Graziano F, Kawakami K, Watanabe G, Santini D, Catalano V, Bisonni R, Canestrari E, Ficarelli R, Menichetti ET, et al: Pharmacogenetic profiling and clinical outcome of patients with advanced gastric cancer treated with palliative chemotherapy. J Clin Oncol 24: 1883-1891, 2006.

40. Sohn KJ, Croxford R, Yates Z, Lucock M and Kim YI: Effect of the methylenetetrahydrofolate reductase C677T polymorphism on chemosensitivity of colon and breast cancer cells to 5-fluorouracil and methotrexate. J Natl Cancer Inst 96: 134-144, 2004

41. Kawakami K, Omura K, Kanehira E and Watanabe G: Methylenetetrahydrofolate reductase polymorphism is associated with folate pool in gastrointestinal cancer tissue. Anticancer Res 21: 285-289, 2001.

42. Mohrenweiser HW, Xi T, Vázquez-Matías J and Jones IM: Identification of 127 amino acid substitution variants in screening 37 DNA repair genes in humans. Cancer Epidemiol Biomarkers Prev 11: 1054-1064, 2002

43. Marusyk A and Polyak K: Tumor heterogeneity: Causes and consequences. Biochim Biophys Acta 1805: 105-117, 2010.

44. Giessen C, Laubender RP, Fischer von Weikersthal L, Schalhorn A, Modest DP, Stintzing S, Haas M, Mansmann UR and Heinemann V: Early tumor shrinkage in metastatic colorectal cancer: Retrospective analysis from an irinotecan-based randomized first-line trial. Cancer Sci 104: 718-724, 2013.

45. Heinemann V, Modest D, von Weikersthal LF, Decker T, Kiani A, Vehling-Kaiser U, Al-Batran SE, Heintges T, Lerchenmüller C, Kahl C, et al: Independent radiological evaluation of objective response, early tumor shrinkage and depth of response in FIRE-3 (AIO KRK-0306). Annals Oncol 25: ii117, 2014.

46. Cui LH, Shin MH, Kweon SS, Kim HN, Song HR, Piao JM, Choi JS, Shim HJ, Hwang JE, Kim HR, et al: Methylenetetrahydrofolate reductase $\mathrm{C} 677 \mathrm{~T}$ polymorphism in patients with gastric and colorectal cancer in a Korean population. BMC Cancer 10: 236, 2010.

47. Kim HN, Kim NY, Yu L, Kim YK, Lee IK, Yang DH, Lee JJ, Shin MH, Park KS, Choi JS and Kim HJ: Polymorphisms of drug-metabolizing genes and risk of non-Hodgkin lymphoma. Am J Hematol 84: 821-825, 2009. 